\title{
Jonathan Swift y su contribución científica en "Los viajes de Gulliver"
}

\author{
MARCELO MIRANDA C. ${ }^{1}$, CAROLINA PÉREZ J., ${ }^{1, a}$, \\ ANDREA SLACHEVSKY CH. ${ }^{2,3,4,5}$
}

\section{Jonathan Swift's scientific contribution on his "Gulliver's Travels"}

Jonathan Swift is one of the most celebrated satirist writers in literature. His well-known "Gulliver's Travels", apart from being a serious human being criticism, contains many interesting and unrecognized comments about medical and other scientific facts. Swift made what seems to be the first account of a dementing illness in the elderly; his description fits well with what we now know as Alzheimer's disease. He also described a condition now called synaesthesia, a very interesting phenomenon that may contribute to creativity. In this paper, we review aspects of his life and reveal these amazing descriptions as samples of Swift's extraordinary power of observation.

(Rev Med Chile 2011; 139: 395-399).

Key words: Alzheimer type senile dementia; Historiography; Medicine in literature.

"Cuando un genio verdadero aparece, lo puedes conocer por este signo: todos los necios conspirarán contra él."

\section{J. Swif.}

T onathan Swift (1667-1745), escritor irlandés, quien fue uno de los más celebrados y mordaces críticos de su época, es recordado por su obra maestra "Los viajes de Gulliver" (1726). En este libro hace mención de innovadores conceptos científicos que destacaremos en este artículo y que han sido muy poco difundidos. Para sus contemporáneos, Swift destacó además por ser un agudo crítico de la política y las relaciones entre Inglaterra e Irlanda, y un importante líder espiritual de la catedral de San Patricio en Dublín, su ciudad natal ${ }^{1,2}$. En este artículo revisaremos algunas de las principales contribuciones científicas de Swift, haciendo un paralelo con su biografía.

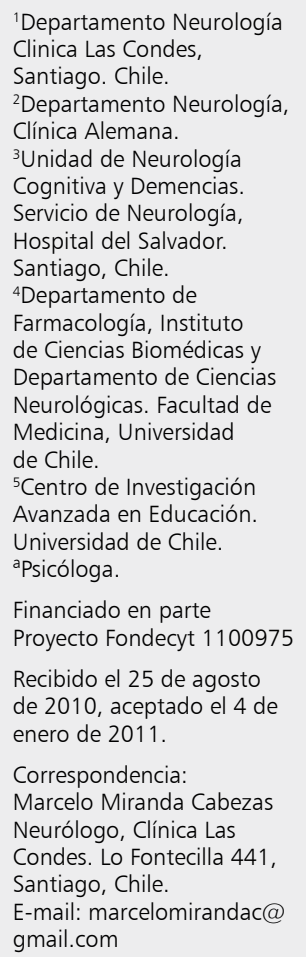


de las corrupciones de los políticos, los vicios y defectos del ser humano, la injustificación de la guerra y sus bajas motivaciones ${ }^{1-5}$.

Esta obra tuvo gran influencia en escritores posteriores, y ha sido adaptada al teatro y cine, traducida a decenas de idiomas y sin lugar a dudas forma parte del acervo cultural de la humanidad.

Jonathan Swift (Figura 1) nació el 30 de noviembre de 1667 en Dublín, Irlanda, en el seno de una familia protestante de origen británicoirlandés. Tuvo una infancia difícil y solitaria, tanto por circunstancias familiares como de salud. Su madre, Abigail Herrick (1642-1710), quedó viuda antes de que él naciera, ya que su padre, Jonathan Swift (1645-1667), un modesto abogado, murió sorpresivamente, sin causa clara, 7 meses antes que naciera su hijo ${ }^{1,6}$.

Durante su infancia, Swift fue muy enfermizo, desarrollando precozmente la que se conocería en 1861 como la enfermedad de Méniere. Los signos y síntomas comenzaron a manifestarse desde los 21 años con vértigo, náuseas y pérdida de la audición. El escritor se referirá a esta enfermedad repetidamente en sus cartas ${ }^{7-9}$.

Después de morir su padre, la madre de Jonathan quedó en una situación de gran pobreza, sin embargo, junto a la niñera hicieron todo lo posible para proporcionarle lo necesario. En sus primeros años quedó al cuidado de familiares de esta última en Inglaterra ${ }^{1}$. A los cuatro años regresó a Irlanda para vivir con su tío paterno Godwin Swift, quien lo apoyó y le facilitó la mejor educación posible. A la edad de 14 años ingresó al famoso Trinity College, en Dublín. Se dice que Jonathan no fue muy feliz en ese período y no logró sobresalir en sus estudios. Es probable que el hecho de no haber conocido a su padre y el escaso contacto con su madre contribuyeran a la animadversión y al carácter crítico e irónico que lo caracterizó con las figuras de autoridad $d^{3,4}$.

En 1688 volvió a Inglaterra para trabajar como secretario de Sir William Temple (1628-1699) en Surrey. A los 22 años conoció al amor de su vida, de sólo 6 años en ese momento, Esther "Stella" Johnson (1680-1728), hija menor de un empleado de Temple. Tuvieron una profunda y larga relación afectiva, Swift se convirtió en su amigo, tutor y mentor ${ }^{1,9}$.

Gracias a las influencias de Temple, en 1692 ingresó a la Universidad de Oxford a estudiar Teología. Un tanto desmotivado de su trabajo

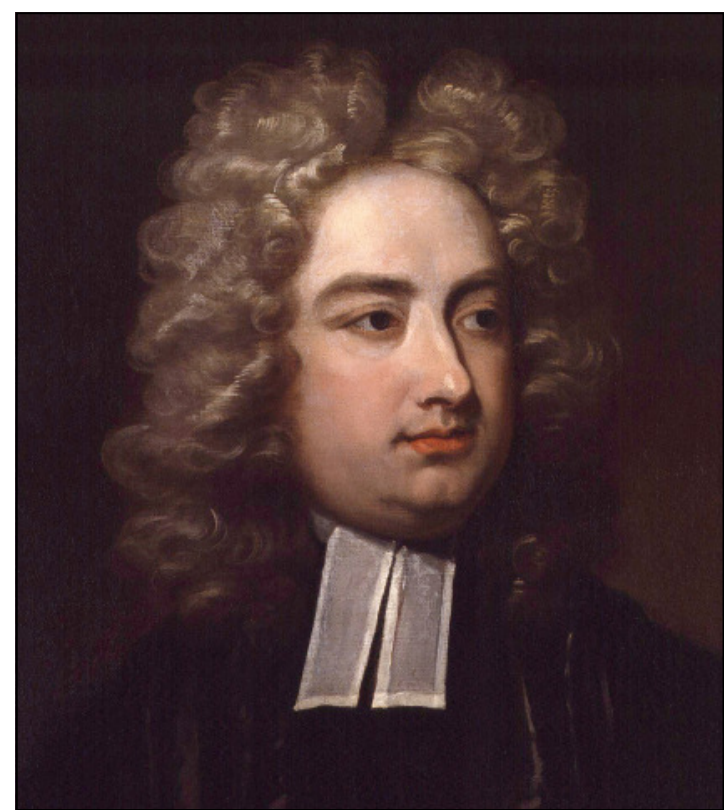

Figura 1. Swift en un retrato de Charles Jervas de fecha desconocida ${ }^{13}$

junto a Temple, volvió a Dublín donde en 1694 fue ordenado pastor anglicano. En 1701 fue nombrado Doctor en Filosofía del Trinity College de Dublín. En 1713 fue elegido Déan de la Catedral de San Patricio en su ciudad natal. En esta época conoció otro amor, Esther "Vanessa" Vanhomrigh (1688-1723), quién le inspiró el poema "Cadenus and Vanessa" (1713). Vanessa, nombre que Swift inventó y que desde entonces se incorporó a nuestro lenguaje, era 22 años menor. En el poema Swift dice: "Cada niña, cuando complacida con lo que se le enseña, tendrá al profesor en sus pensamientos" 2 . En 1723 Swift rompió la relación. Vanessa nunca se recuperó de este rechazo.

Va volviéndose cada vez más satírico, y en obras como "A Modest Proposal" (1729) sugirió con grotesca e irónica lógica que para remediar la pobreza en Irlanda, los niños irlandeses pobres sean usados para alimentar a los ricos ingleses!:

"..Se me ha confirmado por un amigo americano en Londres que un niño bien nutrido es un alimento excelente, delicioso y nutritivo ya sea cocido, al horno o la plancha para los refinados paladares ingleses"

El deterioro cognitivo de Swift y la primera descripción de la demencia tipo Alzheimer en "Los Viajes de Gulliver".

En enero de 1728 muere Esther "Stella" John- 


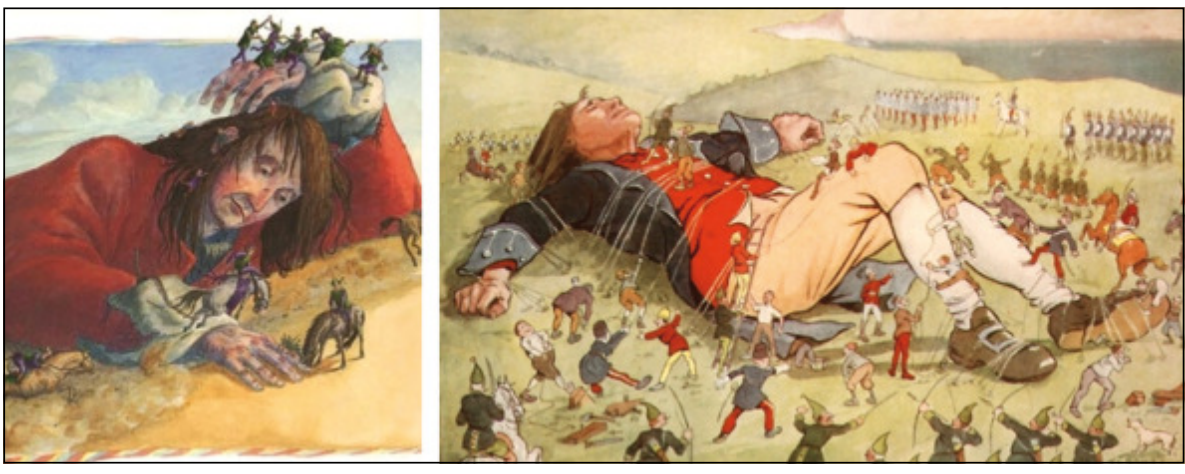

Figura 2 A y B. Característica representación de la llegada de Gulliver a Liliput $^{13}$. son y Swift, se muestra profundamente afectado con gran tristeza, pérdida de la motivación y aislamiento que le durará siempre. Sus últimos 4 años de vida los pasó en melancolía, además de evidenciar progresivas fallas de memoria y defectos de lenguaje. Un familiar escribió sobre él: "algunas veces no pronuncia ni una sílaba, o sólo palabras incoherentes, está como en un estado de completa insensibilidad, duerme mucho y cuesta mucho hacerlo caminar en su habitación". No reconocía a sus amigos $^{2,5,7,8}$. Según Lewis ${ }^{10}$, los síntomas de Swift pueden atribuirse a una demencia tipo Alzheimer ya que los principales cambios experimentados por Swift fueron : i) cambio de memoria; ii) trastorno del comportamiento y iii) pérdida de autonomía. De hecho, 5 meses antes de escribir su testamento, una corte juzgó a Swift incapaz de preocuparse de si mismo. Varios connotados escritores ingleses como Walter Scott y Samuel Johnson dieron a entender que Swift enloqueció en sus últimos años con grandes arrebatos de ira y mayor inadecuación en su trato 6 . Sin embargo, al no conocerlo directamente, ya que no fueron contemporáneos, se cree que malinterpretaron las opiniones de los conocidos de Swift ${ }^{2,7}$.

En los "Versos sobre la muerte del Deán Swift escritos por él mismo" en 1731, 14 años antes de su muerte, dijo:

"Ha perdido su talento para la poesía

Ahora requiere una hora para encontrar una rima... Ve como el Deán comienza a quebrarse

Pobre caballero, pierde el equilibrio,

Tu puedes encontrarlo en su rostro;

ese viejo vértigo en su cabeza nunca lo dejará hasta su muerte;

además, su memoria decae, no recuerda nada de lo que dice" .
Falleció de lo que parece ser, por los antecedentes ya mencionados, una demencia tipo Alzheimer, el 19 de octubre de $1745^{2,5,7,8}$. Se le realizó una necropsia encontrándose lo que se describe inespecíficamente como "un cerebro con edema" restos descansan con los de "Stella" en la Catedral de San Patricio en Dublín.

Swift había escrito su propio epitafio, traducido libremente del latín por su compatriota y premio Nobel de literatura William Butler Yeats:

"Swift ha navegado hacia su descanso. La indignación salvaje no puede allí dañar su corazón. Imítalo si te atreves, viajero amante del mundo. El luchó por la libertad de los hombres"1,2.

"Los Viajes de Gulliver" (1726) (Figuras 2 y 3), es la obra más lograda de Swift, quizás con cierta influencia del "Robinson Crusoe" de Defoe que había aparecido en 1719. En este libro, Swift presenta como protagonista a Lemuel Gulliver, cirujano y

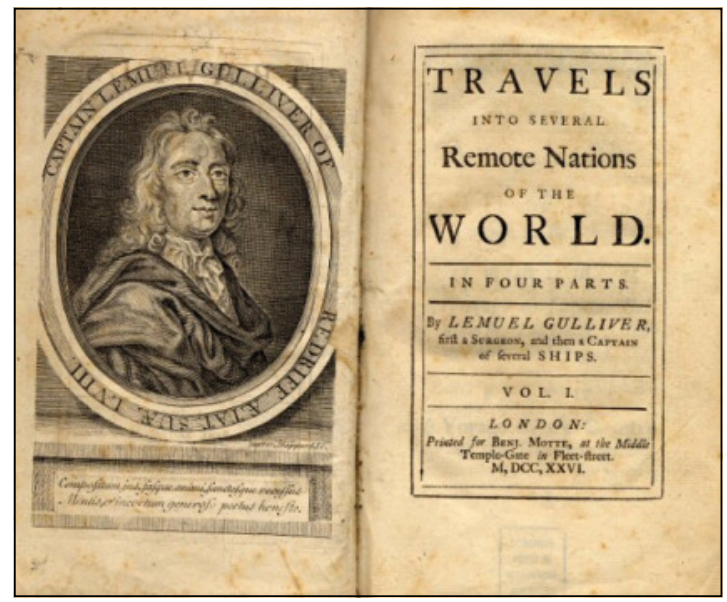

Figura 3. La primera edición de "Los Viajes de Gulliver" en $1726^{13}$. 
capitán de barco, quien hace un recuento de sus aventuras. En la primer parte, Gulliver naufraga en una isla donde los seres humanos tienen una estatura de no más de $12 \mathrm{~cm}$. Estos liliputienses pasan en guerras, y se comportan en forma irrisoria con su arrogancia y vanidad: es decir, presenta las debilidades humanas a menor escala solamente. El segundo viaje lo llevó a Brobdingnag, en cuyo lugar al contar sobre su país le responden: "tus compatriotas son la raza más perniciosa de seres odiosos que la naturaleza alguna vez toleró que se arrastraran por la tierra". En el tercer viaje, se ridiculiza a científicos, mostrando a la ciencia como algo fútil sino es para el beneficio humano. En la isla de Glubbdubdrib Gulliver conoce a los Struldbrughs, seres inmortales. Al respecto hace la siguiente descripción en la cual claramente está relatando lo que hoy conocemos como la Enfermedad de Alzheimer ${ }^{5,10}$ : “... cuando llegan a los 80 años es evidente que al hablar ellos olvidan el nombre de las cosas y el de las personas, incluso aquellos más cercanos y parientes. Por la misma razón no se entusiasman con lo que leen, debido a que la memoria no les ayuda a recordar lo que recién han leído al terminar apenas una oración. Observarlos es mortificante en verdad". "Además son incapaces de distinguir sabores y olores, por lo cual comen y beben sin fruición ni apetito. No recuerdan cosa alguna que no hayan aprendido en su mocedad o en edad madura, e incluso estos recuerdos son borrosos. Los menos desgraciados de ellos parecen aquellos que, víctimas de debilidad senil, pierden la memoria enteramente, pues se les trata con mayor compasión y se les ayuda más"... "más allá de la tercera generación ya no reconocían a su descendencia"... no estaban más en condición de cultivar su mente y de adornar su memoria; y a mayor edad repetían lo dicho recién una y otra vez, habian perdido del todo la memoria y estaban reducidos al estado de infancia y perdían su autonomía”.

En suma, Swift describe personas que asociado a la edad presentan un trastorno de memoria de naturaleza progresiva. Es evidente que Swift con su agudo poder de observación traspasó a sus escritos lo que había observado en la realidad sobre el decaimiento intelectual de las personas; en particular los cambios cognitivos de su protector, su tío Godwin quién murió demenciado ${ }^{7}$.

\section{Una primera descripción de sinestesia}

Sinestesia puede definirse como una "percep- ción multisensorial derivada de sólo una estimulación sensorial" ${ }^{11}$. Ejemplos son la evocación de colores al oír sonidos o ver figuras geométricas al degustar sabores. Es un fenómeno que se asocia a la creatividad y la han manifestado artistas como Nabokov, Rimsky-Korsakov, Kandinsky, ToulouseLautrec, etc. En los viajes de Gulliver, éste llega a un país llamado Laputa y visita una academia llamada Lagado. Entre las cosas sorprendentes que ahí observó, Gulliver describió: "Había un hombre nacido ciego, y quién tenia varios aprendices ciegos como él; el empleo de estos aprendices era mezclar colores para los pintores, tarea que habían aprendido de su maestro logrando distinguir los colores por el tacto y el olfato"3.

\section{Una predicción astronómica}

Gulliver en uno de sus viajes conoció a astrónomos en la isla Laput; Swift describió en ese capítulo que los astrónomos habrían descubierto que Marte tendría 2 satélites o lunas. Swift incluso dio en forma increíble una aproximación bastante cercana a la verdad sobre la distancia que se encuentran aquellas lunas de ese planeta: “... La más interna dista del centro del planeta 3 veces su diámetro, y cinco la más externa; la primera gira en el espacio de 10 horas y la otra en 21.5 horas; así pues los cuadrados de su periodicidad están muy próximos a los cubos de su distancia del centro de Marte. Esto demuestra que están regidas por la misma ley de gravedad que influye sobre los demás cuerpos celestes" ${ }^{3}$. Este hecho recién se describió 150 años después, en 1877, ¿cómo es posible que Swift afirmara esto?, ¿mera coincidencia? No hay una explicación clara a menos que Swift conociera la conjetura similar pero menos completa que Kepler había hecho un siglo antes.

\section{Su interés por la locura}

Desde temprano en sus escritos, Swift mostró interés por la locura ironizando que era muy importante en la comunidad británica y argumenta que sólo es un pensamiento antisocial ${ }^{2}$. Dijo: "si los modernos entienden la locura como un trastorno o transposición del cerebro por influencia de vapores que vienen de niveles inferiores, entonces ha sido esta locura la madre de todas las revoluciones que 
han sucedido en el imperio, en filosofía y en religión. Porque el cerebro en su estado natural de serenidad predispone a su dueño a pasar su vida en forma tranquila, sin pretender subyugar a las multitudes a su propio poder, razonamiento o visiones y mientras más él forma su experiencia y entendimiento por medio del conocimiento humano, menos está inclinado a formar partidos con sus ideas particulares. Ya que el conocimiento lo instruye en sus propias debilidades y en la pertinaz ignorancia del pueblo"12.

En otra carta dice: "las espadas son las lenguas de los locos, y las lenguas son las espadas de los locos". En una "Disgresión sobre la Locura" (1710) dice: "Me gustaría saber cómo dar cuenta de aquellas imaginaciones como las de ciertos hombres en particular, sin recurrir a la teoría de vapores que vienen de niveles inferiores y enturbian el cerebro $y$ alli cristalizan en conceptos o ideas, para lo cual nuestra lengua nativa no tiene otro nombre que no sea locura"12.

Swift hizo referencia en muchos poemas a la presencia de "trastornados mentales o locos" en base a sus frecuentes visitas a sanatorios en Londres y participó en vida en la formación de varios asilos para ellos. Llegó a legar toda su fortuna de 12.000 libras para fundar el primer Hospital Psiquiátrico en Irlanda que se inauguró póstumamente en 1757 y que aún existe ${ }^{7}$.

Con su particular ironía respecto a la locura de los moralmente (y políticamente) corruptos y los realmente enfermos, dice: “...se necesita poder tener un lugar para los locos, truhanes, fastidiosos, garabateros, infieles y mentirosos, sin mencionar a los incurablemente vanidosos, orgullosos, afectadosy otros 10.000 más sin cura". Sugería que él también fuera ingresado como un "garabatero (en el sentido de escribir descuidadamente) incurable"2.

Con su sarcasmo característico, siempre fue crítico de los médicos y su incapacidad para mejorar a los enfermos. En "Los Viajes de Gulliver" y en sus cartas hace claras referencias a la falta de eficacia de la medicina. En este mal concepto, es evidente que influyó el fracaso de tratamiento de los síntomas que sugieren sufría de un severo síndrome de Meniére.
En suma, Jonathan Swift, que destacó por su constante sátira, indudablemente contribuyó a lo que se propuso y que era crear conciencia de los defectos de la humanidad para intentar mejorarla, y ser un catalizador de la libertad de Irlanda. En sus obras, además, especialmente en "Los Viajes de Gulliver", hizo relevantes observaciones científicas muy acertadas y vigentes.

Agradecimientos: A Osvaldo Gil, Bibliotecario de la Facultad de Medicina, Universidad de Chile por su gran ayuda en obtener referencias.

\section{Referencias}

1. Aitken GA. Swift en: Award AW, Waller AR, Trent WP, Erskine J, Herman E (Eds) The Cambridge history of English and American literature, GP Putnam Sons. New York Vol 11; 9: 2000.

2. Lorch MP. Explorations of the Brain, Mind and Medicine in the writings of Jonathan Swift. En Whitaker H, Smith C, Finger S (Eds) Brain, Mind and Medicine: Essays in Eighteen Century Neuroscience. Springer, New York, 2007; 345-52.

3. Swift J. Los viajes de Gulliver. Aguilar. Madrid 1955.

4. Swift J. Obras Escogidas. Austral Summa. Madrid 2007.

5. Lorch JM. Language and memory disorder in the case of Jonathan Swift. Brain 2006; 129: 3127-37.

6. Johnson S. Jonathan Swift. En: Vida de Poetas Ingleses. Cátedra, Madrid 1988.

7. T. G. Wilson. The mental and physical Health of Dean Swift. Med Hist 1958; 2: 175-90.

8. Bewley TH. The Health of Jonathan Swift. J R Soc Med 1998; 91: 602-5.

9. Banks JT. The writ "de lunatico inquirendo" in the case of Jonathan Swift, D. D., Dean of St. Patrick's; with Observations. Dublin Quarterly Journal of Medical Science, 1861; 31: 83-90.

10. Lewis JM. Jonathan Swift and Alzheimer's disease. Lancet 1993; 342: 504.

11. A Larner. A possible account of synaesthesia dating from the seventeenth century. J Hist Neurosci 2006; 15: 245-9.

12. Swift J. Thoughts on various subjects http://e.text. library.adelaide.edu.au/swift/jonathan/s97th/.

13. http://es.wikipedia.org/wiki/Jonathan_Swift. 